\title{
2263. Sparse decomposition based on ADMM dictionary learning for fault feature extraction of rolling element bearing
}

\author{
Qingbin Tong', Zhanlong Sun², Zhengwei Nie', Yuyi Lin ${ }^{4}$, Junci Cao ${ }^{5}$ \\ 1,2,3,5School of Electrical Engineering, Beijing Jiaotong University, Beijing 100044, China \\ ${ }^{3,4}$ Department of Mechanical and Aerospace Engineering, University of Missouri, \\ Columbia MO 65211, USA \\ ${ }^{1}$ Corresponding author

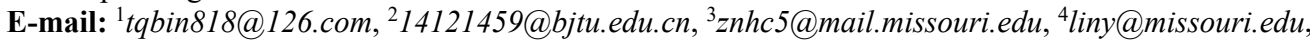 \\ 5jccao@bjtu.edu.cn
}

Received 14 August 2016; received in revised form 30 October 2016; accepted 8 November 2016

DOI https://doi.org/10.21595/jve.2016.17566

\begin{abstract}
Sparse decomposition is a novel method for the fault diagnosis of rolling element bearing, whether the construction of dictionary model is good or not will directly affect the results of sparse decomposition. In order to effectively extract the fault characteristics of rolling element bearing, a sparse decomposition method based on the over-complete dictionary learning of alternating direction method of multipliers (ADMM) is presented in this paper. In the process of dictionary learning, ADMM is used to update the atoms of the dictionary. Compared with the K-SVD dictionary learning and non-learning dictionary method, the learned ADMM dictionary has a better structure and faster speed in the sparse decomposition. The ADMM dictionary learning method combined with the orthogonal matching pursuit (OMP) is used to implement the sparse decomposition of the vibration signal. The envelope spectrum technique is used to analyze the results of the sparse decomposition for the fault feature extraction of the rolling element bearing. The experimental results show that the ADMM dictionary learning method can updates the dictionary atoms to better fit the original signal data than K-SVD dictionary learning, the high frequency noise in the vibration signal of the rolling bearing can be effectively suppressed, and the fault characteristic frequency can be highlighted, which is very favorable for the fault diagnosis of the rolling element bearing.
\end{abstract}

Keywords: sparse decomposition, alternating direction method of multipliers (ADMM), dictionary learning, orthogonal matching pursuit (OMP), K-SVD, rolling element bearing, fault feature extraction.

\section{Introduction}

Rolling element bearings are regarded as one of the most common components in rotating machinery of modern industry. The failure of rolling element bearings can result in the deterioration of machine operating conditions after a long-term running in the complex and severe conditions such as high speed, heavy load, strong impact or high temperature environment $[1,2]$. Therefore, reliable bearing fault detection techniques are very significant to recognize a bearing defect at its earliest stage so as to prevent machinery performance degradation and malfunctions. Bearing fault detection can be undertaken using different information carriers such as vibration signals, lubricant information, and acoustic and temperature data [3]. Among them, vibration signals carry rich condition-related information due to the fact that a series of impact impulses will occur when a rolling element bearing hits a localized fault $[4,5]$. Therefore, vibration-based analysis is mostly commonly applied in the condition monitoring and fault diagnosis of rolling element bearings [6-8]. Nevertheless, in practice the defect-induced impulses are often too weak to be distinguished in the complex data corrupted by a large amount of background noise. Therefore, it is critical to denoise the raw measured signals and extract intrinsic transient characteristics for the fault diagnosis of rolling element bearing at early stages.

To effectively extract the fault feature from the vibration signals, various techniques have been 
developed for the fault diagnosis of rolling element bearing, such as Wigner-Viller distribution (WVD) [9], the wavelet transform (WT) [10], the empirical mode decomposition (EMD) [11, 12], the local mean decomposition (LMD) $[13,14]$, etc. However, traditional methods based on orthogonal linear transforms are not suitable for the multiple components present in the natural complex vibration signals. Sparse representations of signals have received a great deal of attentions in recent years for the fault diagnosis of rolling element bearing [15-17]. Different from the traditional orthogonal basis transformation, the problem solved by the sparse representation is to search for the most compact representation of a signal in terms of linear combination of atoms in an over-complete dictionary [18]. Sparse representation can be served as the decomposition and reconstruction problems. Sparse decomposition mainly consists of two aspects: one type focus on the algorithm optimization and improvement for representing the signal by learned sparse components or sparse atoms, and the other type is on the atom function modeling for an overcomplete dictionary construction. Therefore, successful application of a sparse decomposition depends on the dictionary used, and whether it matches the signal features [19]. At present, there are two main ways to determine an over-complete dictionary in the sparse decomposition: the traditional fixed dictionary and the dictionary learning. The traditional fixed dictionary entails a pre-existing dictionary, such as the Fourier basis, wavelet basis or constructing a dictionary which reflects different properties of the signal. Because these dictionaries are fixed, they cannot be adapted to transform according to the decomposed signal, only suitable for matching the characteristics of specific signals, and achieve the sparse representation of the specific signal [19]. Dictionary learning, on the other hand, aims at deducing the dictionary from the training data, so that the atoms directly capture the specific features of the signal or set of signals. The dictionary learning method is an effective way to solve the problem of the fixed dictionary. Aharon et al. [20] proposed an over-complete dictionary design method. It is essentially a generalization of the K-means clustering. It uses singular value decomposition (SVD) to update dictionary, hence termed K-SVD. The algorithm has been shown to work well in image compression and one dimensional signal processing. However, every dictionary update must be implemented with the SVD algorithm in K-SVD dictionary learning. When the size of dictionary becomes larger, the $\mathrm{K}-\mathrm{SVD}$ algorithm will spend a long time, which is not conducive to the real-time processing of the signal.

In order to effectively extract the fault characteristics of rolling element bearing, on the basis of considering algorithm optimization and improvement for representing the signal by learned sparse components or sparse atoms, an over-complete dictionary learning method based on ADMM dictionary learning is introduced in this paper. The ADMM dictionary learning method combined with the orthogonal matching pursuit (OMP) is used to implement the sparse decomposition of the bearing vibration signal. The envelope spectrum technique is used to analyze the results of the sparse decomposition. Simulation experiments and real experiments are given for verifying the validity of the ADMM dictionary learning and the fault feature extraction method. The rest of the paper is organized as follows. In Section 2, the sparse representation is introduced, while the basic principle of orthogonal matching pursuit is described in Section 3. In Section 4, the ADMM dictionary learning method is proposed. Section 5 will present the experimental results and analysis. Finally, the conclusion is drawn in Section 6.

\section{Sparse representation of signal}

The sparse representation of a signal $f$ is a linear combination of a few elements (atoms) in a given dictionary. Given a dictionary $D \in R^{n \times k}$ that contains $k$ atoms as column vectors $x_{i} \in R^{n}$, $i=1,2, \ldots, k$, a signal $f \in R^{n}$ can be represented as a sparse linear combination of these atoms $[20,21]$. The representation of $f$ can also be expressed as finding the sparsest vector $a \in R^{k}$ such that $f=D a$. Therefore, the problem is to solve the following optimization problem: 
$\min _{a}\|a\|_{0}$, s.t. $\|f-D a\|_{2} \leq \varepsilon$,

where $\varepsilon$ is the reconstruction error of the signal $f,\|a\|_{0}$ is the $\ell_{0}$-norm and is equivalent to the number of non-zero components in the vector $a$.

\section{The basic principle of orthogonal matching pursuit}

Finding the solution of the Eq. (1) is a NP-hard problem due to its nature of combinational optimization [18]. Therefore, a lot of research has been done on algorithms to seek an approximate solution. Matching pursuit algorithms (MP) introduced by Mallat [15] is the greedy algorithms that optimize approximations by selecting dictionary vectors one by one. A shortcoming of the MP algorithm is that if the vertical projection of the residual signal is non orthogonal to the selected atoms, although asymptotic convergence is guaranteed, the resulting approximation after any finite number of iterations will in general be suboptimal. Aiming at the defect of MP, Pati et al. [22] proposed the orthogonal matching pursuit (OMP). The improvement of OMP algorithm is that the selected atoms are carried out by the orthogonal processing at the decomposition step, which makes the convergence rate of the OMP algorithm more quickly in the same accuracy requirements.

Assume $f \in R^{n}$ is the decomposed signal vector, $D=\left\{x_{i}\right\} \in R^{n \times k}$ is the super-complete dictionary, and the columns of $D$ are normalized so that $\left\|x_{i}\right\|_{2}=1, i=1,2, \ldots, k . R^{k} f$ is the residual signal of $k$ th iteration. Initialize $f_{0}=0, R^{0} f=f, D_{0}=\{\}, x_{0}=0, a_{0}^{0}=0, k=0$. Assume the $k$-step decomposition, the signal $f$ is decomposed as follows:

$f=\sum_{n=1}^{k} a_{n}^{k} x_{n}+R^{k} f=f_{k}+R^{k} f, \quad\left\langle x_{n}, R^{k} f\right\rangle=0, \quad n=1,2, \ldots, k$,

where $a_{n}^{k}$ is the coefficients of $k$-step decomposition. the signal $f$ of the $(k+1)$-step decomposition can be given:

$$
\begin{aligned}
& f=\sum_{n=1}^{k+1} a_{n}^{k+1} x_{n}+R^{k+1} f, \quad\left\langle x_{n}, R^{k+1} f\right\rangle=0, \quad n=1,2, \ldots, k+1, \\
& x_{k+1}=\sum_{n=1}^{k} b_{n}^{k} x_{n}+\gamma_{k}, \quad\left\langle\gamma_{k}, x_{n}\right\rangle=0, \quad n=1,2, \ldots, k,
\end{aligned}
$$

where $\sum_{n=1}^{k} b_{n}^{k} x_{n}=P_{V_{k}} x_{k+1}$ represents the projection of $x_{k+1}$ at $\left\{x_{1}, x_{2}, \cdots, x_{k}\right\}, \gamma_{k}=P_{V_{k}^{\perp}} x_{k+1}$ denotes the component of $x_{k+1}$ perpendicular to $\left\{x_{1}, x_{2}, \cdots, x_{k}\right\}$ :

$a_{n}^{k+1}=a_{n}^{k}-\alpha_{k} b_{n}^{k}, \quad n=1,2, \ldots, k, \quad a_{n+1}^{k+1}=\alpha_{k}$.

where:

$a_{k}=\frac{\left\langle R^{k} f, x_{k+1}\right\rangle}{\left\langle\gamma_{k}, x_{k+1}\right\rangle}=\frac{\left\langle R^{k} f, x_{k+1}\right\rangle}{\left\|\gamma_{k}\right\|^{2}}=\frac{\left\langle R^{k} f, x_{k+1}\right\rangle}{\left\|x_{k+1}\right\|^{2}-\sum_{n=1}^{k} b_{n}^{k}\left\langle x_{n}, x_{n+1}\right\rangle}$.

The residual signal $R^{k+1} f \quad$ satisfies $\quad R^{k} f=R^{k+1} f-\alpha_{k} \gamma_{k}$, and $\left\|R^{k+1} f\right\|^{2}=\left\|R^{k} f\right\|^{2}-\left\langle R^{k} f, x_{k+1}\right\rangle^{2} /\left\|\gamma_{k}\right\|^{2}$. The specific steps of the OMP algorithm can be described as follows [22]:

Step 1: Compute $\left\{\left\langle R^{k} f, x_{n}\right\rangle ; x_{n} \in D \backslash D_{k}\right\}$. 
Step 2: Find $x_{n}^{k+1} \in D \backslash D_{k}$ such that $\left|\left\langle R^{k} f, x_{n}^{k+1}\right\rangle\right| \geq \alpha \sup _{j}\left|\left\langle R^{k} f, x_{j}\right\rangle\right|, 0<\alpha \leq 1$.

Step 3: If $\left|\left\langle R^{k} f, x_{n}^{k+1}\right\rangle\right|<\delta,(\delta>0)$ then stop.

Step 4: Reorder the dictionary $D$, by applying the permutation $k+1 \leftrightarrow n_{k+1}$.

Step 5: Compute $\left\{b_{n}^{k}\right\}_{n=1}^{k}$, such that $x_{k+1}=\sum_{n=1}^{k} b_{n}^{k} x_{n}+\gamma_{k}$, and $\left\langle\gamma_{k}, x_{n}\right\rangle=0, n=1,2, \ldots, k$.

Step 6: Set $a_{k+1}^{k+1}=a_{k}=\left\|\gamma_{k}\right\|^{\perp 2}\left\langle R^{k} f, x_{k+1}\right\rangle, a_{n}^{k+1}=a_{k}-a_{k} b_{n}^{k}, n=1,2, \ldots, k$, and update the mode $f_{k+1}=\sum_{n=1}^{k+1} a_{n}^{k+1} x_{n}, R^{k+1} f=f-f_{k+1}, D_{k+1}=D_{k} \cup\left\{x_{k+1}\right\}$.

Step 7: Set $k \leftarrow k+1$, and repeat the step 1-7.

\section{The proposed ADMM dictionary learning method}

\subsection{The alternating direction method of multipliers}

The alternating direction method of multipliers (ADMM) is a powerful algorithm for solving structured convex optimization problems $[23,24]$. By constructing the augmented Lagrangian, ADMM algorithm can be used to split the objective function of the original problem into several low dimensional sub-problems which are easy to find the local solution for the iterative solution, so as to get the global solution of the original problem.

The ADMM algorithm solves problems of the form:

$\min f(x)+g(y)$, s.t. $A x+B y=c$,

where $f$ and $g$ are convex functions, $x \in R^{n}, y \in R^{m}, A \in R^{p \times n}, B \in R^{p \times m}$ and $b \in R^{p}$.

The augmented Lagrangian of the Eq. (2) is:

$L(x, y, \lambda)=f(x)+g(y)+\lambda^{T}(A x+B y-c)+\left(\frac{\rho}{2}\right)\|A x+B y-c\|_{2}^{2}$,

where $\rho>0$ is penalty parameter, Lagrange multiplier is $\lambda \in R^{p}$.

The iterative scheme of ADMM for the Eq. (6) is:

$\left\{\begin{array}{l}x^{k+1}=\operatorname{argmin}_{x} L_{\rho}\left(x, y^{k}, \lambda^{k}\right), \\ y^{k+1}=\operatorname{argmin}_{z} L_{\rho}\left(x^{k+1}, y, \lambda^{k}\right), \\ \lambda^{k+1}=\lambda^{k}+\rho\left(A x^{k+1}+B y^{k+1}-c\right) .\end{array}\right.$

It can be seen from the Eq. (8) that the iterative steps of the ADMM algorithm include the minimization $x$ and $y$, and a dual variable iteration step. In this algorithm, $x$ and $y$ are iteratively updated, and then the dual variable $\lambda$ is updated iteratively. The iterative scheme of ADMM embeds a Gaussian-Seidel decomposition into each iteration of the augmented Lagrangian method (ALM); thus, the functions $f$ and $g$ are treated individually and so easier sub-problems could be generated. This feature is very advantageous for a broad spectrum of application.

\subsection{ADMM dictionary learning method}

In the sparse decomposition of the bearing vibration signal, it is very important to construct a good dictionary. Although the fixed dictionary structure is redundant, the atoms are not necessarily consistent with the physical properties of the decomposed signal, and cannot be adaptive adjusted according to the signal, so the results of signal decomposition may not be ideal. The dictionary obtained by learning is more consistent with the characteristics of the decomposed signal, and can get a better decomposition effect in the process of sparse decomposition. The dictionary is implemented the learning process according to the decomposed signal, so that it can better fit the physical properties of the decomposed signal, and can get more sparse decomposition coefficient, 
get better decomposition results than the non-dictionary learning.

The dictionary learning in the sparse decomposition of bearing vibration signals can be represented as:

$\min _{D, X}\left\{\|Y-D X\|_{F}^{2}\right\}, \quad$ s.t. $\quad\left\|x_{i}\right\| \leq k, \quad i=1,2, \ldots, L$,

where $Y$ is the training matrix, $D$ is the dictionary, $X$ denotes the projection of the signal onto the dictionary $D, k$ is the upper bound of the sparsity coefficients.

The Eq. (9) is implemented the optimization approximations based on ADMM dictionary learning. First, based on the given initial dictionary $D$ and training matrix $Y$, the OMP algorithm is used to implement the sparse coding for solving the coefficient $X$. Then, fix coefficient $X$, update the dictionary $D$ using the dictionary learning. According to the steps mentioned above, the iteration is done until the given of iteration times are reached or satisfies the error requirement of the signal reconstruction. In the process of dictionary learning based on ADMM algorithm, the Eq. (9) is firstly converted to the following format:

$\min _{D, X, Z}\left\{\|Y-D X\|_{F}^{2}\right\}, \quad$ s.t. $Z=D X, \quad\left\|x_{i}\right\|_{0} \leq k$.

Therefore, the Lagrange function of dictionary learning can be obtained:

$L=\|Y-Z\|_{F}^{2}+\sum_{i=1}^{L}\left\langle\Lambda_{i},(Z-D X)_{i}\right\rangle+\frac{\beta}{2}\|Z-D X\|_{F}^{2}$,

where $\Lambda$ is Lagrange multiplier matrix, $\Lambda_{i}$ denote the $i$ th column of $\Lambda$.

The ADMM algorithm is applied to the Eq. (11), and the OMP algorithm is used to solve the coefficients of the equation, and finally get the updated dictionary:

$D^{(n+1)}=D^{(n)}(:, i)+\frac{H^{(n)} X^{(n)}(:, i)^{T}}{\omega^{(n)}+\delta}$.

The ADMM dictionary learning algorithm can be stated as follows.

Step 1: Initialize the dictionary $D^{0}$, this matrix can be a matrix $m \times n$ of random distribution, and also are the column vectors $m$ with the length $n$ chosen from a given signal. The Lagrange multiplier matrix is $\Lambda^{0}$. The sparsity and iteration times are $k$ and $K$, respectively. Two positive numbers are $\alpha$ and $\beta$.

Step 2: Main loop: determine the number of loops according to the given update error.

Step 3: Sparse decomposition: Using the OMP algorithm to solve the coefficient matrix $X$ :

$X=O M P(D, Y, k)$.

Step 4: Update dictionary:

$$
\begin{aligned}
& G^{(n)}=\frac{\beta D^{(n)} X^{(n)}+2 Y-\Lambda^{(n)}}{2+\beta}, \\
& H^{(n)}=G^{(n)}+\frac{\Lambda^{(n)}}{\beta}-D^{(n)} X^{(n)} .
\end{aligned}
$$

Step 5: Sub-loop:

$\omega^{(n)}=X^{(n)}(:, i) X^{(n)}(:, i)^{T}$, 
$D^{(n+1)}=D^{(n)}(:, i)+\frac{H^{(n)} X^{(n)}(:, i)^{T}}{\omega^{(n)}+\delta}$.

Step 6: The dictionary $D$ is implemented the normalization processing, and update Lagrange multiplier matrix:

$\Lambda^{(n+1)}=\Lambda^{(n)}+\gamma \beta\left(G^{(n)}-D^{(n+1)} X^{(n)}\right)$.

Step 7: If the iteration reaches the specified times or satisfies the error requirement of the signal reconstruction, stop the algorithm. Otherwise, return to Step 3.

The selection of parameter $\beta$ and the matrix $\Lambda$ have a certain effect on the convergence of the dictionary update in the dictionary learning, they can be adjusted according to the need of the specific experiment.

\section{Experimental analysis and discussion}

\subsection{Simulation analysis using proposed dictionary learning}

In order to verify the advantages of the proposed method in dictionary learning and random signal reconstruction, a simulation experiment is designed and carried out. The random signal is a random sparse signal of normal distribution generated by the function Sprandn(). Fig. 1 shows the generated random signal. Firstly, the random signals are used to carry out the dictionary learning and the sparse decomposition. The training matrix $Y$ is a matrix $m \times p$ of random generation in the experiment. In order to ensure the effectiveness of the dictionary learning, take $p=5 \mathrm{~m}$. The matrix with the size $m \times n$ generated by the random is used as the initial matrix $D$, where $n=2 m$, and each column of the matrix is implemented the normalization process. In order to compare the performance of different methods in dictionary learning, the fixed iteration numbers (10 times) and the same sparsity $(k=15)$ are selected. The dictionary learning methods of ADMM and K-SVD are respectively carried out the dictionary learning, and record the learning time of the two methods. When the size of the dictionary is changed, the running speed of the two methods is compared.

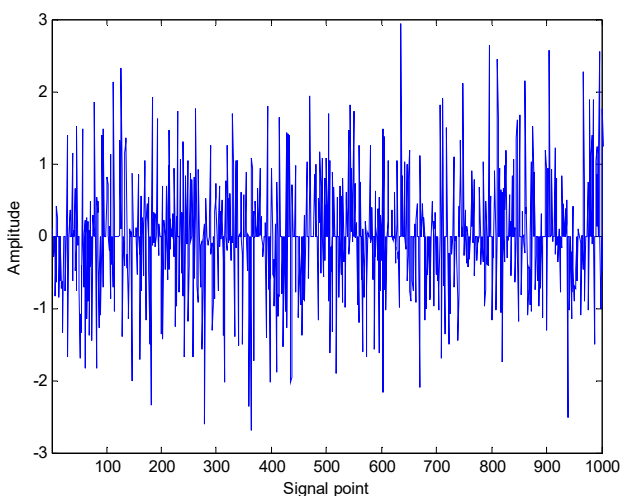

Fig. 1. Random signal generated by function $\operatorname{Sprandn}()$

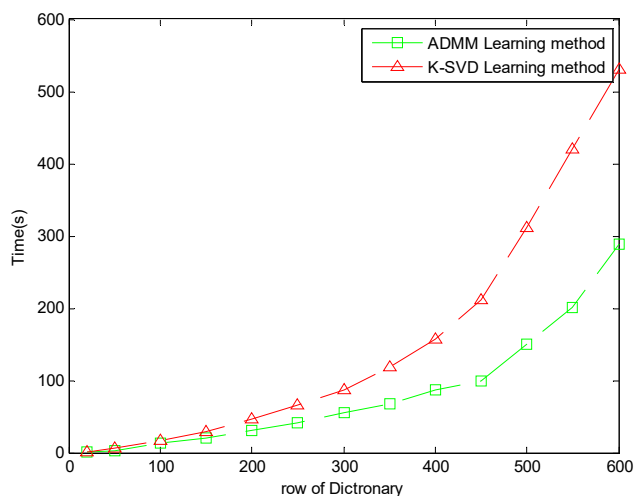

Fig. 2. Compare of learning time in different dictionary size

Fig. 2 shows the learning time of the dictionary row numbers from 50 to 600 , the horizontal axis in Fig. 1 is the column numbers of the dictionary training, the vertical axis is the needed time of the learning process. The specific running time is given in Table 1. As shown in Fig. 2, it is clear that the running time of the ADMM dictionary learning is less than the K-SVD dictionary learning method in the same size of the testing matrix, dictionary and the iteration number. And 
with the increasing of the size of the dictionary, this advantage is more and more obvious. When the size of the dictionary is 600, the learning time of ADMM method is almost half of K-SVD method.

In order to further verify the superiority of the proposed method, the simulation signal is simulated as follows:

$s(t)=2 \cos (2 \pi f t+5)+v$,

where $v$ is the random noise of standard normal distribution, the signal-to-noise ratio is $S_{N R}=-10 \mathrm{~dB}$. Fig. 3 shows the waveform of the simulation signal.

Table 1. Specific time of dictionary learning

\begin{tabular}{|c|c|c|}
\hline \multirow{2}{*}{ Size of dictionary } & \multicolumn{2}{|c|}{ Learning time (s) } \\
\cline { 2 - 3 } & ADMM & K-SVD \\
\hline 50 & 3.25 & 5.72 \\
\hline 100 & 12.44 & 17.40 \\
\hline 200 & 31.33 & 45.74 \\
\hline 300 & 55.23 & 87.08 \\
\hline 400 & 87.00 & 156.8 \\
\hline 500 & 150.56 & 310.81 \\
\hline 600 & 289.34 & 530.23 \\
\hline \multicolumn{2}{|c}{} \\
\hline
\end{tabular}

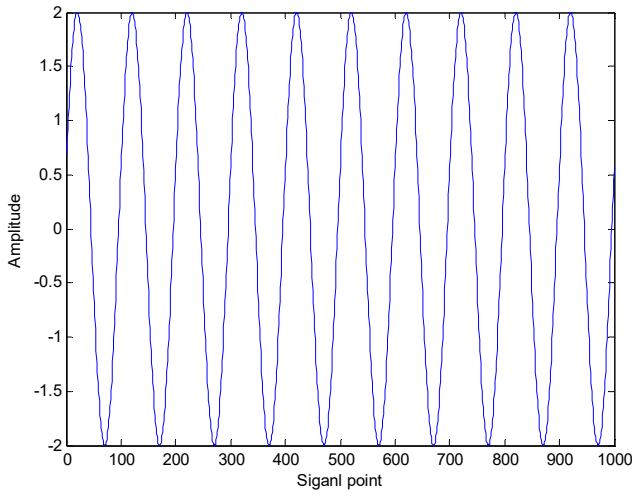

a) Original signal

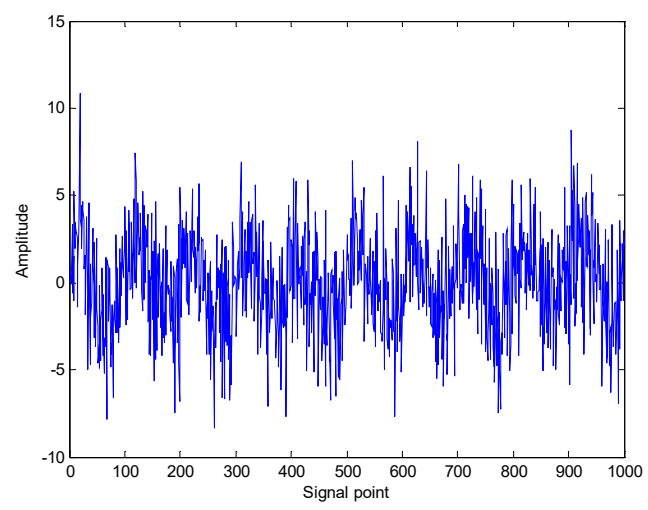

b) Signal with the noise

Fig. 3. The waveform of the simulation signal

The training matrix $Y$ is obtained from the additive noise signal, and the dictionary is regarded as the initial dictionary. The original signal is decomposed by the sparse decomposition. The root mean square error (RMSE) of the reconstructed signal is obtained. The calculation formula of RMSE is as follows:

$R M S E=\sqrt{\left\|I-I_{n}\right\|_{F}^{2} / \text { length }(I)}$,

where $I$ is the original signal, $I_{n}$ is the reconstructed signal. The size of the training matrix is $100 \times 300$, the size of the dictionary is $100 \times 200$, the sparsity of the decomposition is 15 . The RMSE of the reconstructed signal with the change of the iteration number is shown in Fig. 4.

It can be seen from Fig. 4 that the RMSE of the reconstructed signal after dictionary learning is obviously less than the RMSE of without learning, and with the increase of iteration number in the dictionary learning, the RMSE of the reconstructed signal is gradually reduced. The RMSE of ADMM learning dictionary is obviously lower than the value of K-SVD dictionary learning, and 
with the increase of the numbers of iteration, the gap will continue to increase. But when the iteration number is more than 80 , the value of RMSE tends to be stable, which indicates that the effect of signal decomposition is not increased with the increase of the iteration number of the dictionary learning. From the RMSE of the signal reconstruction, the ADMM dictionary learning algorithm is significantly better than the K-SVD dictionary learning.

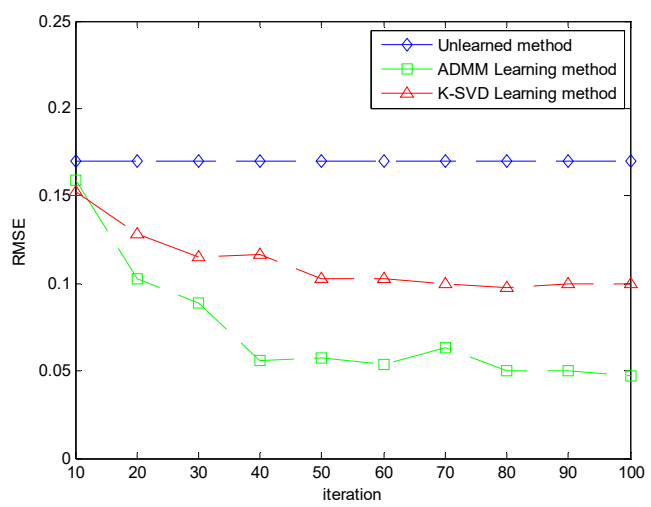

Fig. 4. RMSE of different methods

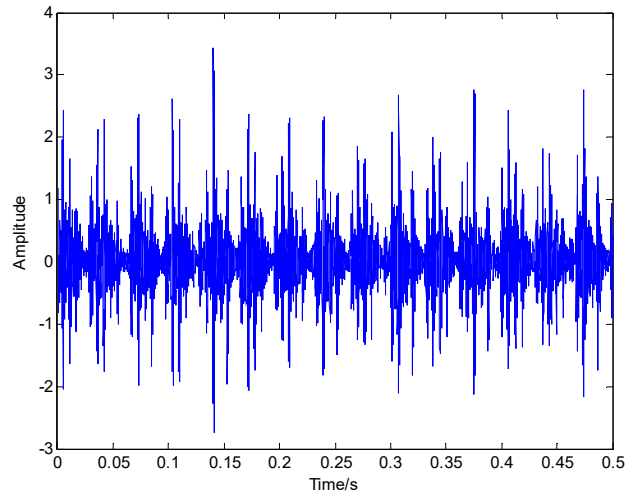

Fig. 5. Time domain plot of inner race defect

\subsection{Analysis of the bearing vibration signal for the fault feature extraction}

In order to verify the effectiveness of the proposed method in the sparse decomposition of bearing vibration signals, the actual experiment on fault identification of rolling element bearings is conducted in this paper. The vibration data of rolling bearings are provided by Case Western Reserve University (CWRU) [25]. The deep groove ball bearing with the type of 6205-2RS JEM SKF was used in the test. The vibration signals, when the rotating speed is $1797 \mathrm{rpm}$, and the sampling frequency is $12 \mathrm{KHz}$, are chosen to extract fault feature in this paper. The characteristic frequency of the inner race defect is calculated to be at $164 \mathrm{~Hz}$, the outer race defect and the rolling element defect is $106 \mathrm{~Hz}$ and $128.9 \mathrm{~Hz}$ respectively based on the geometric parameters. Fig. 5, Fig. 6 and Fig. 7 illustrates the representative waveforms of the signal with the inner race defect, the outer race defect and the rolling element defect, respectively.

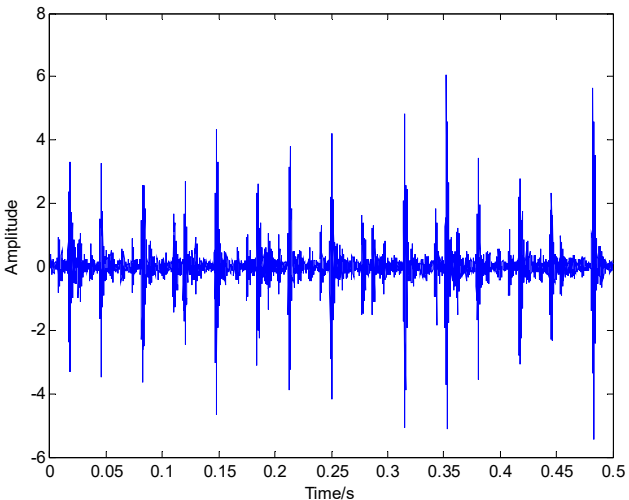

Fig. 6. Time domain plot of outer race defect

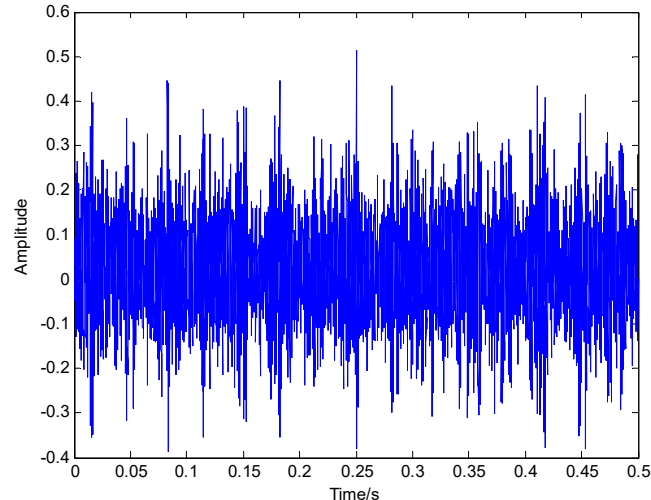

Fig. 7. Time domain plot of rolling element defect signal

In this experiment, the data points of length 30000 are intercepted from the bearing vibration signal, which is used to construct the training matrix of $100 \times 200$, and the data points of length 1000 are intercepted from the remaining signal as the testing signal. The constructed dictionary is used as the dictionary to be learned, and the K-SVD and ADMM are used respectively to carry 
out the dictionary learning. According to the learned dictionary, the test signal is decomposed and reconstructed by using OMP algorithm, and the residual of the reconstructed signal is obtained, and the reconstructed signal is implemented by spectral analysis.
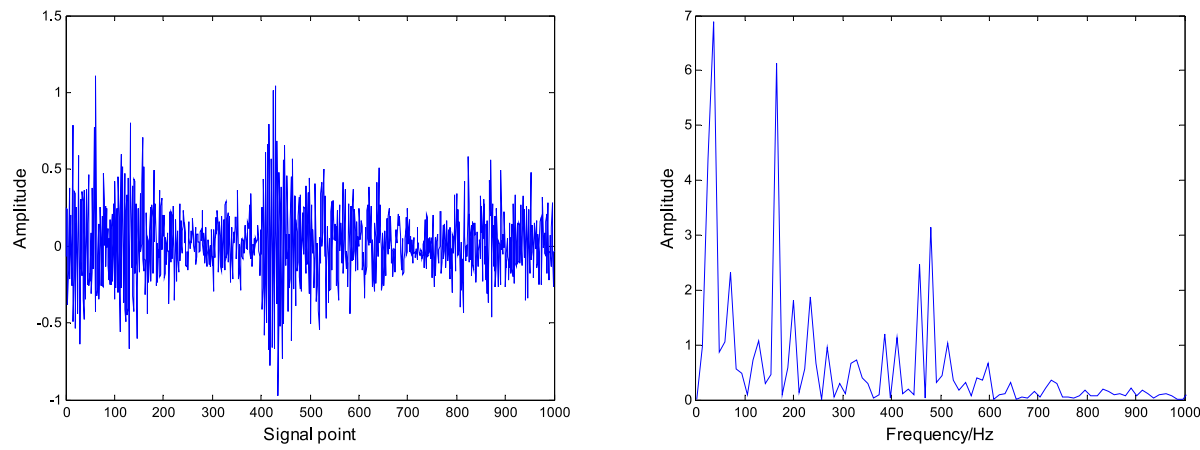

a) Without dictionary learning
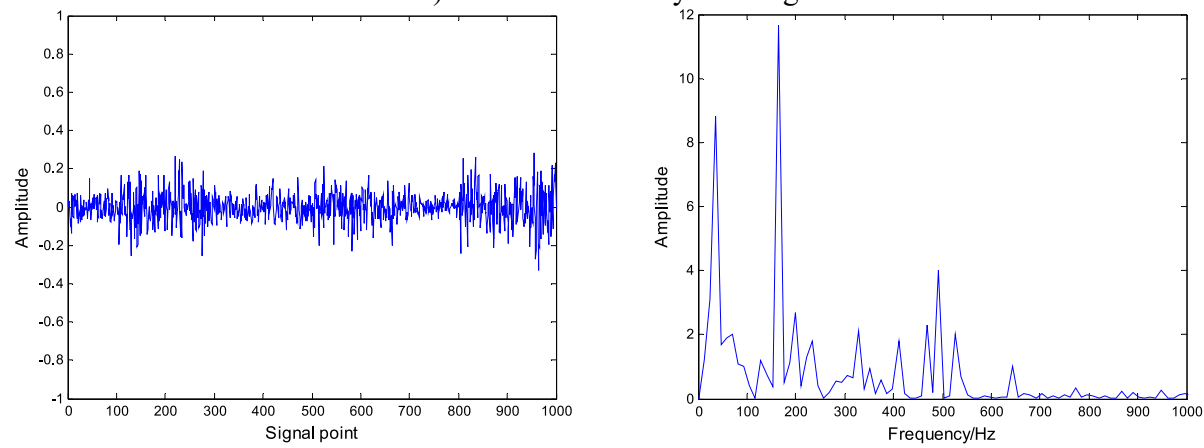

b) K-SVD dictionary learning
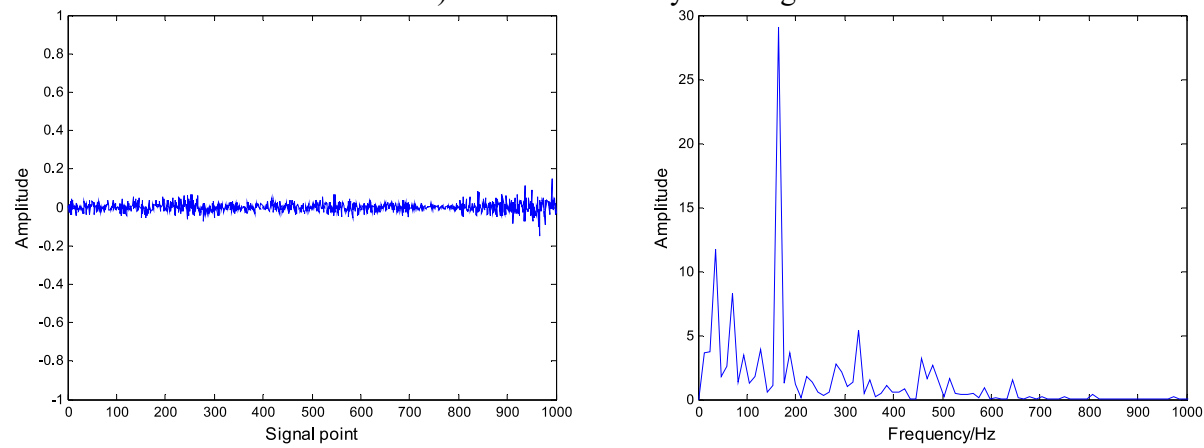

c) ADMM dictionary learning

Fig. 8. Residual and envelope spectrum by spare decomposition for inner race defect

In the case of the same training matrix, the initial dictionary and the number of iterations, the dictionary learning and the sparse decomposition of test signals are implemented for obtaining the envelope spectrum of the reconstructed signal and the residual. When the iteration number of the dictionary learning is 30 , the sparsity of decomposition is 20 , Fig. 8, 9 and 10 show the envelope spectrum of the reconstructed signal and the residual results of the inner race defect, the outer race defect and the rolling element defect by using the related method.

It can be seen that by Figs. 8, 9 and 10, under the same number of iterations and sparsity, the residual amount of reconstruction signal after the dictionary learning is far less than the residual amount of without learning. The residual amount of the proposed ADMM dictionary learning is smaller than that of the K-SVD dictionary learning method, which indicates that the dictionary 
constructed by ADMM dictionary learning is more consistent with the physical characteristics of the decomposed signal, and has better performance in sparse decomposition and reconstruction.
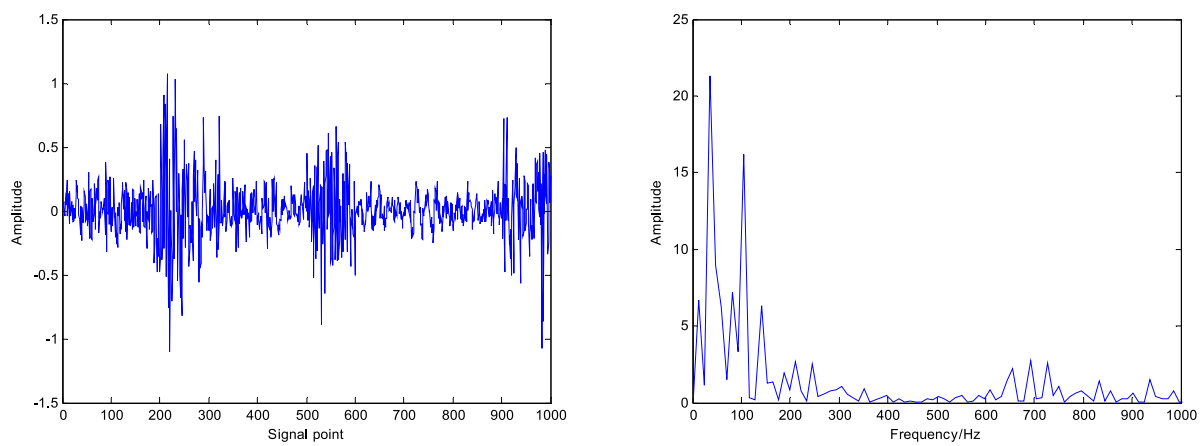

a) Without dictionary learning
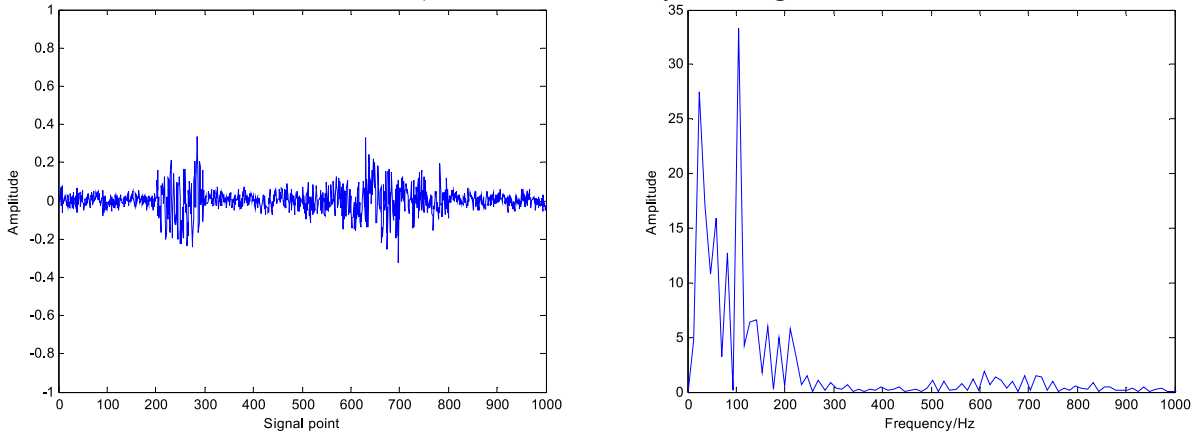

b) K-SVD dictionary learning
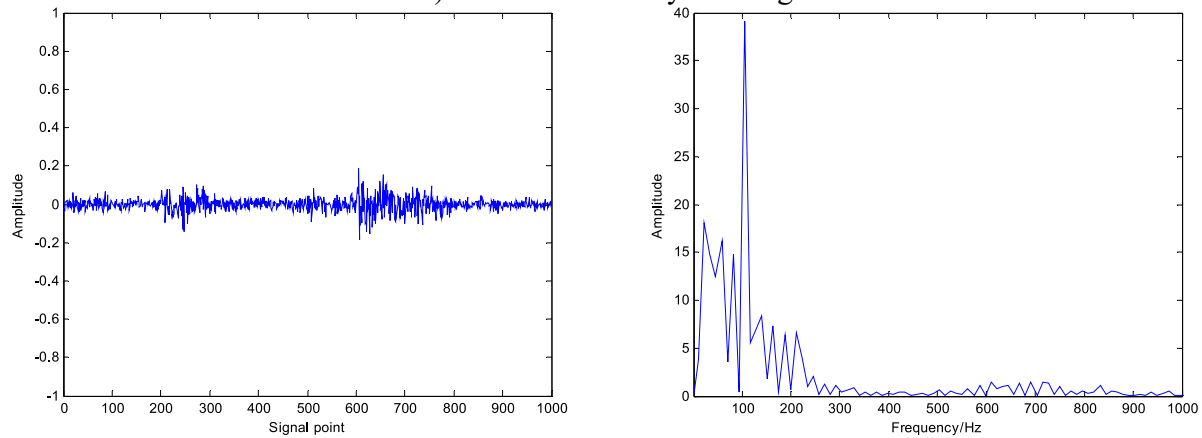

c) ADMM dictionary learning

Fig. 9. Residual and envelope spectrum by spare decomposition for outer race defect

Compared with the envelope spectrum in Figs. 8, 9 and 10, it can be clearly seen that the decomposed effect of dictionary learning is better than that of the non-learning, and the fault frequency of the envelope spectrum is more obvious, and the interference frequency is less. At the same time, under the same condition, the resulting envelope spectrums of bearing fault signal obtained by ADMM dictionary learning and K-SVD dictionary learning are different. In the envelope spectrum of the bearing fault signal obtained by ADMM dictionary learning, the fault frequency of bearing inner race is very obvious. Although there are some interference frequencies, the amplitude is much smaller. However, the fault frequency can be identified in the envelope spectrum of the bearing fault signal obtained by K-SVD dictionary learning, but the amplitude of some interference frequencies is very high. It can be concluded that the dictionary obtained by ADMM dictionary learning is more consistent with the characteristics of the decomposed signal, and can get a better decomposition effect in the process of sparse decomposition. 

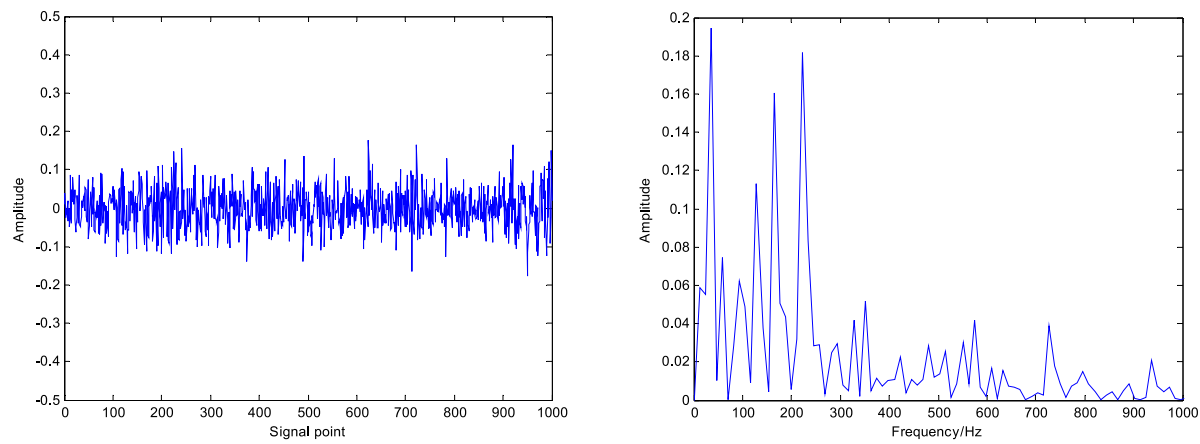

a) Without dictionary learning
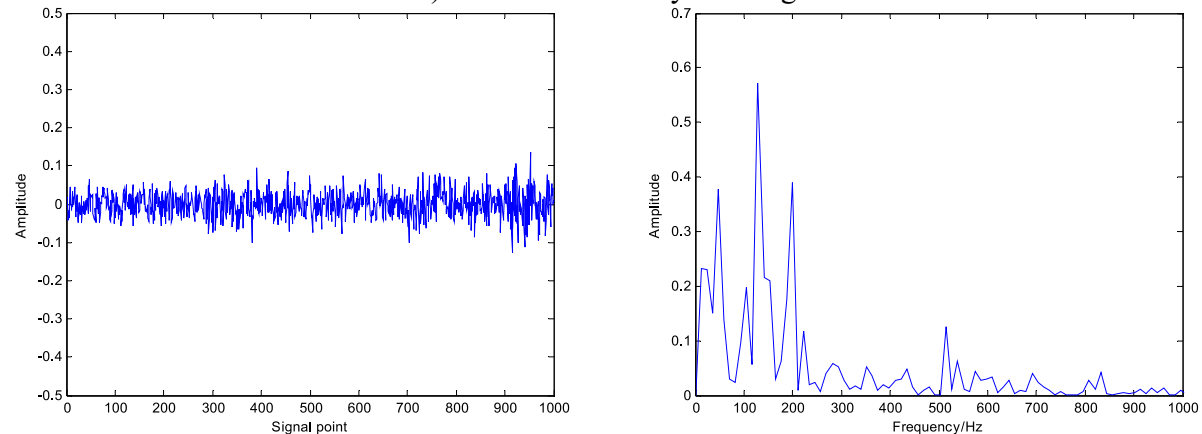

b) K-SVD dictionary learning
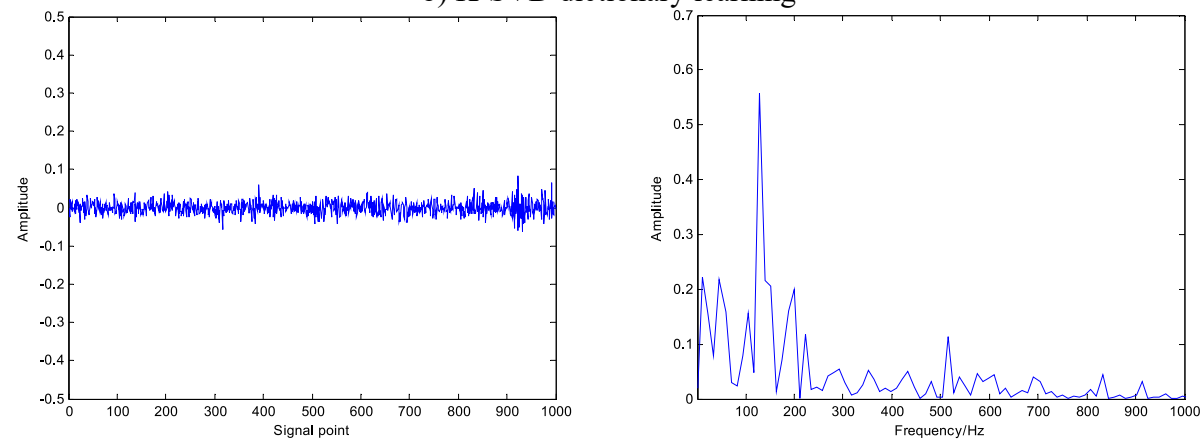

c) ADMM dictionary learning

Fig. 10. Residual and envelope spectrum by spare decomposition for rolling element defect

Qingbin Tong, as the first author and corresponding author, his contribution is the idea of the article, writing and programming. Zhanlong Sun, his contribution is the preparation of article procedures, data analysis. Zhengwei Nie, his contribution is the preparation of article procedures, data analysis. Yuyi Lin, his contribution is to improve the language of the article. Junci Cao, his contribution is to further improve the article and the funding.

\section{Conclusions}

In this paper, a dictionary learning method based on ADMM is presented for obtaining a better dictionary in structure, and the ADMM dictionary learning method combined with the orthogonal matching pursuit (OMP) is used to implement the sparse decomposition of the bearing vibration signal for the fault feature extraction. The experimental results show that this method has a faster speed and better sparse decomposition results. Compared with the K-SVD dictionary learning method, the proposed method has the superiority in the sparse decomposition of bearing signals. The experimental results show that, compared with the fixed dictionary and the K-SVD dictionary 
under the same conditions, the proposed ADMM dictionary learning method has not only fast learning speed, but also better reflect the characteristics of the decomposed signal. The proposed method is used to decompose the vibration signal of the rolling element bearing, the less residual can be obtained, the high frequency noise in the vibration signal of the rolling bearing can be effectively suppressed, and the fault characteristic frequency can be highlighted, which is very favorable for the fault diagnosis of the rolling element bearing.

\section{Acknowledgements}

This study was supported by State Key Laboratory of Alternate Electrical Power System with Renewable Energy Sources (Grant No. LAPS15019), the Fundamental Research Foundations for the Central Universities (Grant No. 2014JBZ017) and the National Science Foundation of China (Grant No. 51577007).

\section{References}

[1] Zhang X., Zhou J. Multi-fault diagnosis for rolling element bearings based on ensemble empirical mode decomposition and optimized support vector machines. Mechanical Systems and Signal Processing, Vol. 41, Issue 1, 2013, p. 127-140.

[2] Qu J., Zhang Z., Gong T. A novel intelligent method for mechanical fault diagnosis based on dual-tree complex wavelet packet transform and multiple classifier fusion. Neurocomputing, Vol. 171, 2016, p. 837-853.

[3] Sui W., Osman S., Wang W. An adaptive envelope spectrum technique for bearing fault detection. Measurement Science and Technology, Vol. 25, Issue 9, 2014, p. 095004.

[4] Jiang F., Zhu Z., Li W., et al. Robust condition monitoring and fault diagnosis of rolling element bearings using improved EEMD and statistical features. Measurement Science and Technology, Vol. 25, Issue 2, 2014, p. 025003.

[5] Lei Y., Lin J., He Z., et al. Application of an improved kurtogram method for fault diagnosis of rolling element bearings. Mechanical Systems and Signal Processing, Vol. 25, Issue 5, 2011, p. 1738-1749.

[6] Liu X., Bo L., He X., et al. Application of correlation matching for automatic bearing fault diagnosis. Journal of Sound and Vibration, Vol. 331, Issue 26, 2012, p. 5838-5852.

[7] Muruganatham B., Sanjith M. A., Krishnakumar B., et al. Roller element bearing fault diagnosis using singular spectrum analysis. Mechanical Systems and Signal Processing, Vol. 35, Issue 1, 2013, p. $150-166$.

[8] Wang W., Lee H. An energy kurtosis demodulation technique for signal denoising and bearing fault detection. Measurement Science and Technology, Vol. 24, Issue 2, 2013, p. 025601.

[9] Mekhilef S. Numerical and experimental analysis of vibratory signals for rolling bearing fault diagnosis. Mechanics, Vol. 22, Issue 3, 2016, p. 217-224.

[10] Peng Z. K., Peter W. T, Chu F. L. A comparison study of improved Hilbert-Huang transform and wavelet transform: application to fault diagnosis for rolling bearing. Mechanical Systems and Signal Processing, Vol. 19, Issue 5, 2005, p. 974-988.

[11] Lei Y., Lin J., He Z., et al. A review on empirical mode decomposition in fault diagnosis of rotating machinery. Mechanical Systems and Signal Processing, Vol. 35, Issue 1, 2013, p. 108-126.

[12] Li Y., Xu M., Wei Y., et al. An improvement EMD method based on the optimized rational Hermite interpolation approach and its application to gear fault diagnosis. Measurement, Vol. 63, 2015, p. $330-345$.

[13] Li Y., Xu M., Haiyang Z., et al. A new rotating machinery fault diagnosis method based on improved local mean decomposition. Digital Signal Processing, Vol. 46, 2015, p. 201-214.

[14] Cheng J., Yang Y., Yang Y. A rotating machinery fault diagnosis method based on local mean decomposition. Digital Signal Processing, Vol. 22, Issue 2, 2012, p. 356-366.

[15] Mallat S. G., Zhang Z. Matching pursuits with time-frequency dictionaries. IEEE Transactions on Signal Processing, Vol. 41, Issue 12, 1993, p. 3397-3415.

[16] He Q., Ding X. Sparse representation based on local time-frequency template matching for bearing transient fault feature extraction. Journal of Sound and Vibration, Vol. 370, 2016, p. 424-443.

[17] Ding X., He Q. Time-frequency manifold sparse reconstruction: a novel method for bearing fault feature extraction. Mechanical Systems and Signal Processing, Vol. 80, 2016, p. 392-413. 
[18] Huang K., Aviyente S. Sparse representation for signal classification. Advances in Neural Information Processing Systems, 2006, p. 609-616.

[19] Jafari M. G., Plumbley M. D. Fast dictionary learning for sparse representations of speech signals. IEEE Journal of Selected Topics in Signal Processing, Vol. 5, Issue 5, 2011, p. 1025-1031.

[20] Aharon M., Elad M., Bruckstein A. SVD: an algorithm for designing overcomplete dictionaries for sparse representation. IEEE Transactions on Signal Processing, Vol. 54, Issue 11, 2006, p. 4311-4322.

[21] Do T. H., Tabbone S., Terrades O. R. Sparse representation over learned dictionary for symbol recognition. Signal Processing, Vol. 125, 2016, p. 36-47.

[22] Pati Y. C., Rezaiifar R., Krishnaprasad P. S. Orthogonal matching pursuit: recursive function approximation with applications to wavelet decomposition. The 27th Asilomar Conference on Signals, Systems and Computers, 1993, p. 40-44.

[23] Boyd S., Parikh N., Chu E., et al. Distributed optimization and statistical learning via the alternating direction method of multipliers. Foundations and Trends in Machine Learning, Vol. 3, Issue 1, 2011, p. 1-122.

[24] Chen C., He B., Ye Y., et al. The direct extension of ADMM for multi-block convex minimization problems is not necessarily convergent. Mathematical Programming, Vol. 155, Issues 1-2, 2016, p. 57-79.

[25] http://csegroups.case.edu/bearingdatacenter.

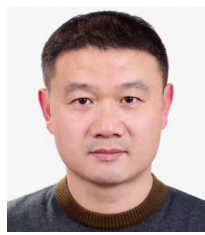

Qingbin Tong received the Ph.D. degree in instrument science and technology from the Harbin Institue of Technology, China, in 2008. He is currently an Associate Professor in the School of Electrical Engineering, Beijing Jiaotong University. His research interests mainly include automotive engineering, fluid transmission and control, engineering applications of artificial intelligence, and mechanical vibration.

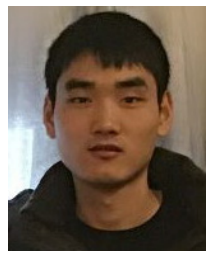

Zhanlong Sun is currently studying for the Master degree in electrical engineering, Beijing Jiaotong University, China, in 2016. His research interests mainly include automotive engineering, engineering applications of artificial intelligence, and mechanical vibration.

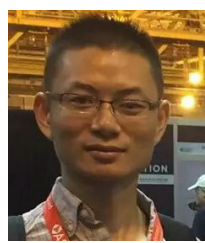

Zhengwei Nie received his Master's degree in power engineering and engineering thermophysics from Xi'an Jiaotong University, Xi'an, China in 2013. He is currently a Doctoral candidate in mechanical engineering at University of Missouri, Missouri, USA. His current research interests include CFD-based optimization and simulation, lightweight materials, fabrication of porous materials, metamaterials, etc.

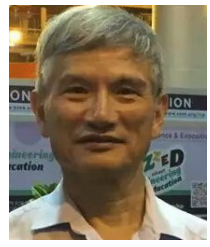

Yuyi Lin received the M.S. degree from the University of California-Los Angeles, USA, and the Ph.D. degree from the University of California-Berkeley, USA, in 1984, and 1989, respectively. Since July 1990, he has been with the Department of Mechanical and Aerospace Engineering, College of Engineering, University of Missouri, USA, where he is currently an Associate Professor. He is a fellow of ASME. His research interests include dynamics and control of mechanisms, optimization, numerical methods, and artificial intelligence techniques.

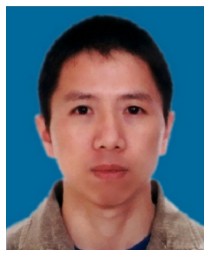

Junci Cao received the B.S., M.S., degrees in Electrical Engineering from Harbin University of Science and Technology, Harbin, China, in 2001, 2004, respectively. He received the $\mathrm{Ph} . \mathrm{D}$. degree in Electrical Engineering from the Harbin Institute of Technology, Harbin, China, in 2008. He is currently an Associate Professor in the School of Electrical Engineering, Beijing Jiaotong University. His research interests mainly include asynchronous traction drive system design, synchronous traction drive system design, simulation and reliability analysis, special motor design and integrated physical field research, motor optimization design and system energy-saving projects. 